\title{
INFLUENCE DU CODE CIVIL CATALAN (2010) SUR LES DÉCISIONS DE GARDE PARTAGÉE. COMPARAISONS ENTRE LA CATALOGNE ET LE RESTE DE ESPAGNE \\ Montserrat Solsona, Jeroen Spijker
}

Institut national d'études démographiques (INED) | «Population »

2016/2 Vol. 71 | pages 313 à 341

ISSN 0032-4663

ISBN 9782733210666

Article disponible en ligne à l'adresse :

http://www.cairn.info/revue-population-2016-2-page-313.htm

\section{Pour citer cet article :}

Montserrat Solsona, Jeroen Spijker, «Influence du Code civil catalan (2010) sur les décisions de garde partagée. Comparaisons entre la Catalogne et le reste de Espagne », Population 2016/2 (Vol. 71), p. 313-341.

DOI 10.3917/popu.1602.0313

Distribution électronique Cairn.info pour Institut national d'études démographiques (INED).

(C) Institut national d'études démographiques (INED). Tous droits réservés pour tous pays.

La reproduction ou représentation de cet article, notamment par photocopie, n'est autorisée que dans les limites des conditions générales d'utilisation du site ou, le cas échéant, des conditions générales de la licence souscrite par votre établissement. Toute autre reproduction ou représentation, en tout ou partie, sous quelque forme et de quelque manière que ce soit, est interdite sauf accord préalable et écrit de l'éditeur, en dehors des cas prévus par la législation en vigueur en France. Il est précisé que son stockage dans une base de données est également interdit. 


\title{
Influence du Code civil catalan (2010) sur les décisions de garde partagée. Comparaisons entre la Catalogne et le reste de Espagne
}

\begin{abstract}
En Europe, la part de parents séparés qui optent pour la garde partagée des enfants tend à augmenter. Ce mode de résidence après le divorce ou la séparation permet un contact régulier de l'enfant avec chacun de ses parents, un meilleur partage des responsabilités éducatives et réduit le risque de perte de contact avec l'un des parents, souvent observé en cas de résidence exclusive. Dans quelle mesure les lois sur la famille peuvent-elles favoriser ce mode de résidence et infléchir les tendances? En 2010, la Catalogne a mis en place un nouveau volet sur la famille dans son code civil, qui introduit des recommandations pratiques pour les juges et pour les parents, visant à pacifier les relations entre conjoints séparés et à faciliter la mise en place d'une coparentalité après le divorce. S'appuyant sur cet exemple et comparant la Catalogne au reste de l'Espagne à partir de données individuelles sur les jugements de divorce intervenus entre 2007 et 2012, Montserrat SoLSONA et Jeroen SPIJKER analysent les évolutions de la fréquence de la garde alternée et de ses principaux déterminants. Ils discutent également des enjeux légaux et comportementaux de ce nouveau mode de résidence en termes de partage des responsabilités parentales.
\end{abstract}

Depuis les années 1960, les relations intimes et familiales entre les sexes et les générations ont connu dans les sociétés occidentales des transformations profondes marquées par plus de liberté de choix individuel et une plus grande diversité des comportements (Bauman, 2003, 2005 ; Beck et Beck-Gernsheim, 1995; Giddens, 1991 ; Kaufmann, 1993, 2007 ; Lesthaeghe, 1983). L'Espagne, encore soumise à l'époque à un régime fasciste autoritaire allié à l'Église catholique romaine sur les questions de droit de la famille et de droit civil, a suivi

\footnotetext{
* Centre d'Estudis Demografics, Barcelona, Espagne.
}

Correspondance : Montserrat Solsona, Centre d'Estudis Demografics, Edifici E2, Universitat autonoma de Barcelona, 08193 Bellaterra, Espagne, courriel : msolsona@ced.uab.es 
cette tendance plus tardivement ${ }^{(1)}$. Cependant, la mort de Franco a été accompagnée d'un retour à la démocratie, de droits élargis pour les individus et de lois adaptées aux évolutions culturelles en matière de sexualité, de relations et de comportements démographiques, c'est-à-dire les lois sur la contraception (1978), le divorce (1981), l'avortement (1985) et, plus récemment, le mariage entre personnes de même sexe (2005). En outre, le modèle patriarcal qui avait dominé les $\mathrm{XIX}^{\mathrm{e}}$ et $\mathrm{XX}^{\mathrm{e}}$ siècles a été rendu obsolète par les nouvelles pratiques familiales.

Les changements juridiques mentionnés ont légitimé la diversité sexuelle, la rupture du lien marital et le contrôle de la fécondité. Cependant, pour que les nouvelles lois aient un effet concret, il était nécessaire de s'attaquer ouvertement à deux questions de genre : la division sexuelle du travail rémunéré et non rémunéré (comme les soins aux enfants), les relations de pouvoir entre les sexes et les générations. Toutes ces transformations sont intimement liées aux problèmes de responsabilité parentale et de résidence des enfants après une séparation ou un divorce, car elles questionnent le partage équitable des responsabilités et charges éducatives entre les parents. La garde partagée ou résidence alternée, désignant ici une situation où les soins aux enfants sont (plus ou moins) équitablement répartis entre les parents, peut être considérée comme un signe de progrès vers l'égalité des sexes.

Lobjectif principal de cette étude est de déterminer si la législation spécifique de la Catalogne sur la parentalité après le divorce peut expliquer (en partie) la plus forte prévalence de la garde partagée accordée par les juges catalans dans les procédures de divorce.

L'article présente d'abord un résumé de la réforme de 2005 sur le divorce en Espagne et du Code civil catalan (CCC) de 2010 sur la garde partagée. Nous décrivons ensuite comment les accords de garde partagée d'enfants mineurs, tels qu'ils ont été décidés par les juges, évoluent dans le temps et varient en fonction des caractéristiques des individus, du mariage et du divorce, en se basant sur les données individuelles issues des jugements de divorce. Les analyses sont menées sur la période 2007-2012 et les résultats pour la Catalogne sont comparés avec ceux du reste de l'Espagne. Nous concluons en discutant dans quelle mesure la législation catalane peut expliquer les différences territoriales observées en matière de garde partagée.

(1) À l'exception de la période brève de la seconde République (1931-1936) où les idées républicaines françaises d'antimonarchisme et d'anticléricalisme ont inspiré de nombreuses lois sur les relations familiales, notamment la reconnaissance, nouvelle à l'époque, des droits reproductifs. Ces lois étaient similaires à celles mises en œuvre des décennies plus tard dans de nombreux pays d'Europe occidentale, notamment en matière d'avortement et de divorce sur la base du consentement mutuel. Mais lorsque Franco est arrivé au pouvoir, ces avancées juridiques ont été abrogées (Moran, 1995). 


\section{La garde partagée : un concept juridique et une pratique}

Le concept juridique de garde partagée (joint legal custody) peut être envisagé comme un élément régulateur des transitions après une séparation ou un divorce. Concrètement, il cherche à réconcilier les droits individuels des conjoints avec leurs responsabilités parentales. Cela comprend le partage de décisions importantes liées à la santé ou la scolarisation des enfants. Ce type de législation a d'abord été adopté aux États-Unis (Caroline du Nord) en 1957. Entre la fin des années 1970 et les années 1990, des réformes similaires ont eu lieu dans la plupart des autres États américains, ainsi que dans les pays anglosaxons et européens (Escobedo et al., 2011; Folberg, 1991; Graversen, 1986; Halla, 2009; Savolainen, 1986; Spruijt et Duindam, 2009).

En Espagne, cependant, la garde légale conjointe (joint legal custody) n'est pas nommée comme telle, elle s'articule dans la loi sur le divorce de 1981 autour du terme d'« autorité parentale »(patria potestad) ${ }^{(2)}$. Depuis 2010, elle est définie en Catalogne par le CCC comme la « responsabilité parentale». Que les parents soient en union ou séparés, mariés ou dans une relation informelle, ils jouissent de ce droit indépendamment du fait qu'ils aient ou non la garde physique de l'enfant -à moins qu'un jugement ne révoque ce droit pour un parent ou les deux (Ibanez-Valverde, 2004; Solsona et al., 2014).

La garde partagée effective (joint physical custody) fait référence aux arrangements résidentiels décidés par le juge, à savoir le ou les endroits où l'enfant réside durant une période donnée. Du point de vue de la vie quotidienne de l'enfant, la garde partagée a plus d'impact que la garde légale conjointe dans la mesure où elle établit concrètement le partage des soins et où elle permet à l'enfant d'avoir des contacts avec ses deux parents (en particulier les pères). Une exception, qui fait toujours débat, peut exister pour les très jeunes enfants, pour lesquels on a montré que des changements fréquents de résidence peuvent être significativement associés à des problèmes d'insécurité affective. Pour ces très jeunes enfants, la garde exclusive avec des visites régulières de l'autre parent peut être considérée comme plus bénéfique que la garde partagée (Tornello et al., 2013) ${ }^{(3)}$.

Par ailleurs, il est établi que la garde partagée améliore l'estime de soi de l'enfant et facilite l'adaptation après le divorce en améliorant les relations pèreenfant. Elle réduit le stress et la charge parentale, ainsi que les conflits et les risques de remise en cause des décisions judiciaires entre les anciens époux,

(2) La loi espagnole de 1981 sur le divorce permet l'exercice conjoint de l'autorité parentale après un divorce et reconnaît les droits de la mère, historiquement réservés aux seuls pères (Lathrop, 2012).

(3) Plusieurs auteurs mettent en garde à propos de la sélection non aléatoire des différents accords de garde lors de l'interprétation des études de corrélation (Bauserman, 2012; Cheadle et al., 2010; McClain, 2011). Cela est lié au fait que, de manière générale, les pères ayant la garde partagée ont un niveau d'éducation et des revenus plus élevés, des enfants plus âgés au moment de la séparation et de meilleures relations avec leur ex-conjoint et leur enfant. Cela explique pourquoi Tornello et al. (2013) ont principalement enquêté sur les enfants issus de ménages aux revenus modestes. 
ce qui est contraire à la plupart des expériences où le droit de résidence est exclusif - en particulier pour les parents non gardiens (Bauserman, 2012; Turunen, 2015). Le fait qu'aucun des deux parents ne soit menacé par la mise à l'écart de son enfant joue un rôle crucial dans leur bien-être.

Il convient de noter, cependant, que la garde partagée au sens juridique ne coïncide la plupart du temps pas à une symétrie parfaite entre les deux parents en termes de partage des tâches et de temps passé avec les enfants, dans la mesure où cela n'est souvent pas possible. Entre les pays (et dans le cas des États-Unis entre les États) ${ }^{(4)}$, il n'existe pas de définition uniforme (voire pas de définition dans certains cas) de la durée minimale pendant laquelle l'enfant doit vivre avec chaque parent dans le cadre de la garde partagée (Bjarnason et Arnarsson, 2011; Sodermans et al., 2013) ${ }^{(5)}$. C'est également le cas pour l'Espagne et la Catalogne.

\section{Législations espagnole et catalane sur le divorce et la garde partagée}

La rupture légale du mariage ${ }^{(6)}$ en Catalogne et dans le reste de l'Espagne est devenue possible grâce à la loi sur le divorce de 1981 (L30/1981), bien qu'elle soit assez restrictive. Par exemple, la loi exige une justification pour rompre un mariage. Avant la fin du Xx ${ }^{\mathrm{e}}$ siècle, la multiplication des séparations et des divorces a commencé à mettre l'appareil judiciaire sous pression, ce qui s'est inévitablement traduit par une réforme juridique. La réforme sur le divorce de 2005 élimine la nécessité d'une séparation judiciaire préalable au divorce ainsi que la présentation d'une cause de divorce. Cela a mené à une chute importante des séparations de couples mariés et à l'accélération des divorces (qui représentent $95 \%$ de toutes les dissolutions de mariages en 2012, contre $40 \%$ en 2006; figure 1) ${ }^{(7)}$. Cependant, avec le début de la diminution du nombre total de mariages quelques années auparavant, le rapport entre le nombre de dissolution de mariages et le nombre de mariages est resté à peu près constant depuis 2006.

(4) Aux États-Unis, on utilise également le terme de garde physique partagée (shared physical custody) pour signifier que les deux parents disposent de périodes «significatives » ou « substantielles » de garde physique, afin de garantir à l'enfant des contacts fréquents et continus avec les deux parents (Buehler et Gerard, 1995; Cancian et Meyer, 1998).

(5) Pas plus qu'il n'existe un critère commun pour définir la garde partagée ou la résidence alternée dans la recherche (Kitterød et Lyngstad, 2012). Cela affecte la prévalence de la garde partagée en fonction des déclarations, la proportion ayant tendance à augmenter si la définition de ce type d'accord sur la résidence des enfants est plus large (Masardo, 2009).

(6) La réforme sur le divorce de 2005 fournit également le cadre juridique pour les séparations d'unions non mariées, c'est le cas en Catalogne depuis la loi de 1998 sur les unions de cohabitants stables (Llei 10/1998 d'unions estables de parella).

(7) L'Institut national de la statistique espagnol ne collecte pas de données sur les dissolutions d'unions non maritales (voir également note 13). 
Figure 1. Mariages, séparations d'unions mariées et divorces, Espagne, 1980-2012

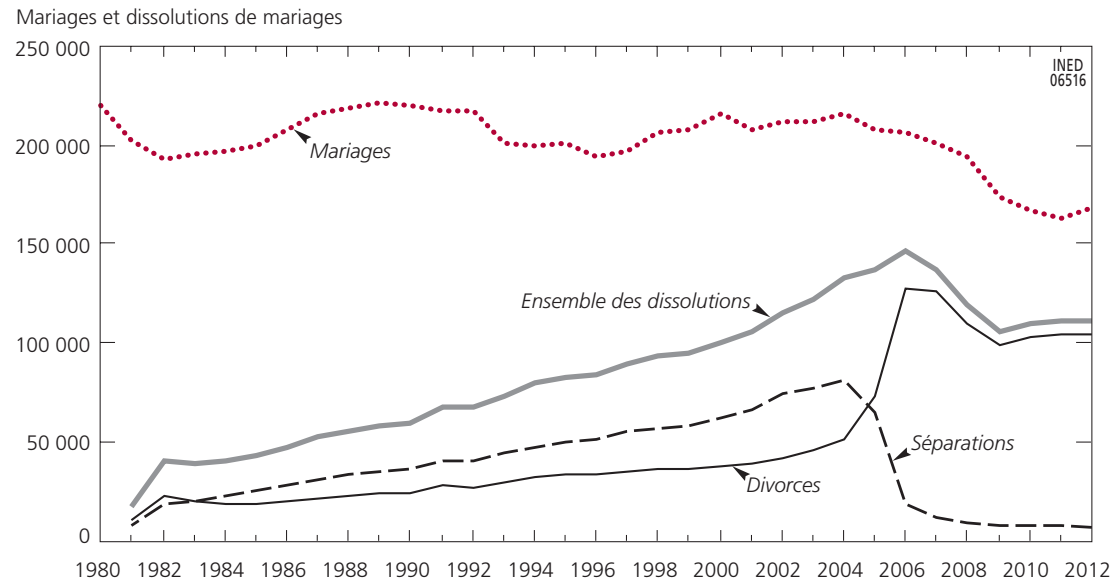

Source : www.ine.es

\section{La réforme espagnole sur le divorce (Loi 15/2005)}

Notre étude s'intéresse particulièrement au fait que la réforme espagnole sur le divorce prévoit explicitement la possibilité, parallèlement à la garde individuelle, d'assigner juridiquement la garde partagée aux deux parents afin que les enfants puissent vivre alternativement avec la mère et le père. Auparavant, ce type d'arrangements résidentiels se pratiquait de manière informelle tant que les parents étaient d'accord, mais il était rarement choisi. La nouvelle législation espagnole (ainsi que le CCC de 2010) établit non seulement la possibilité juridique d'une parentalité partagée après le divorce, mais elle souligne également la coresponsabilité des parents envers leur enfant. En outre, un fonds a été créé pour garantir le versement de la pension en cas de non-paiement du parent débiteur, et la législation prévoit la possibilité de faire appel à des services de médiation familiale (Simó et Solsona, 2010).

Les modalités des relations entre les époux ainsi que celles de la résidence de leurs enfants sont prises sur la base d'un arrangement à l'amiable (convenio regulador). Même si les deux parents demandent la garde partagée, c'est le juge qui évalue avec précaution le bien-fondé de la demande. Le juge peut demander un rapport du procureur, entendre le mineur si nécessaire, et évaluer les allégations de chaque partie concernant les relations entre époux, et celles entre les époux et l'enfant. De même, dans des circonstances exceptionnelles, le juge peut décider d'attribuer la garde partagée contre le souhait des parents (CatalánFrías, 2011; Solsona et al., 2014).

Si les changements législatifs ont fourni un cadre juridique à la garde partagée, les rôles de genre traditionnels ont continué d'influencer fortement l'attribution exclusive de la garde au profit des mères. Selon Catalán-Frías 
(2011), les pères étaient souvent réticents à demander la garde parce qu'ils estimaient que les femmes étaient mieux préparées ou que leur entourage allait penser qu'ils enlevaient l'enfant à la mère. Par ailleurs, les avocats ont souvent découragé leur client de demander la garde exclusive, étant donné les faibles chances de l'obtenir. Cela s'explique par le fait que, généralement, une attribution de la garde au père sous-entend que la mère est trop paresseuse ou incompétente pour s'occuper des enfants. Des preuves empiriques confirment cette hypothèse : l'analyse de 782 contestations de jugement de divorce entre 1993 et 1999 en Espagne (Arce et al., 2005) montre que l'on demandait plus souvent aux pères qu'aux mères de motiver leur demande en cas de garde exclusive (62 \% pour les pères contre $40 \%$ pour les mères). La demande de résidence exclusive de l'enfant chez le père était aussi basée sur une volonté d'exclure la mère plus souvent que d'exclure le père dans le cas de figure inverse. Néanmoins, durant une conférence d'avocates espagnoles en 2006, il a été souligné que la faible proportion de pères disposant de la garde exclusive n'était pas tant le reflet d'une discrimination dans les tribunaux, mais simplement la conséquence de ce que les pères ne la demandaient pas (Catalán-Frías, 2011).

Lorsque la réforme espagnole sur le divorce a facilité la possibilité de garde partagée des enfants après un divorce ou une séparation, on a pensé que cela améliorerait la continuité de la vie familiale de l'enfant, réduirait les conflits entre les parents et permettrait aux pères de se sentir plus responsables de l'éducation et du développement de leur enfant. On estime qu'à l'époque $2 \%$ des gardes étaient partagées, 5 \% étaient attribuées aux pères et $93 \%$ à la mère (ibid.). En 2007, l'année où les données sur les accords de garde ont été pour la première fois enregistrées par les tribunaux (section III), 9,7 \% des jugements en Espagne attribuaient une garde partagée, ce qui suggère que la nouvelle loi a eu un effet modeste quoique significatif. En 2010, le chiffre n'était que de $10,5 \%$; mais depuis, la garde partagée a progressé régulièrement pour représenter 15,3\% des accords en 2013 (www.ine.es).

Récemment cependant, plusieurs régions espagnoles autonomes, qui détiennent le pouvoir de légiférer sur des questions de droit civil, ont produit leurs propres lois qui se substituent aux lois nationales sur la charge des enfants après une rupture de mariage : l'Aragon (Loi 2/2010), la Catalogne (Loi 25/2010), la Navarre (Loi 3/2011) et la région de Valence (Loi 5/2011). Ces lois vont plus loin que la réforme de 2005 sur le divorce en encourageant une parentalité partagée. La situation en Catalogne est expliquée plus en détail ci-dessous. Alors qu'avant l'application de ces lois, ces régions autonomes affichaient déjà des proportions plus importantes de gardes partagées que dans le reste de l'Espagne, la plupart du temps, les différences n'ont fait que s'amplifier (à l'exception de la Navarre). C'est particulièrement vrai pour l'Aragon et la Catalogne, où plus d'un quart des gardes sont partagées, contre seulement 10,4\% dans le reste de l'Espagne (figure 2). 
Figure 2. Proportion de gardes partagées attribuées aux parents divorcés dans les régions autonomes d'Espagne disposant de leurs propres lois sur la famille (2007-2012) et le reste de l'Espagne

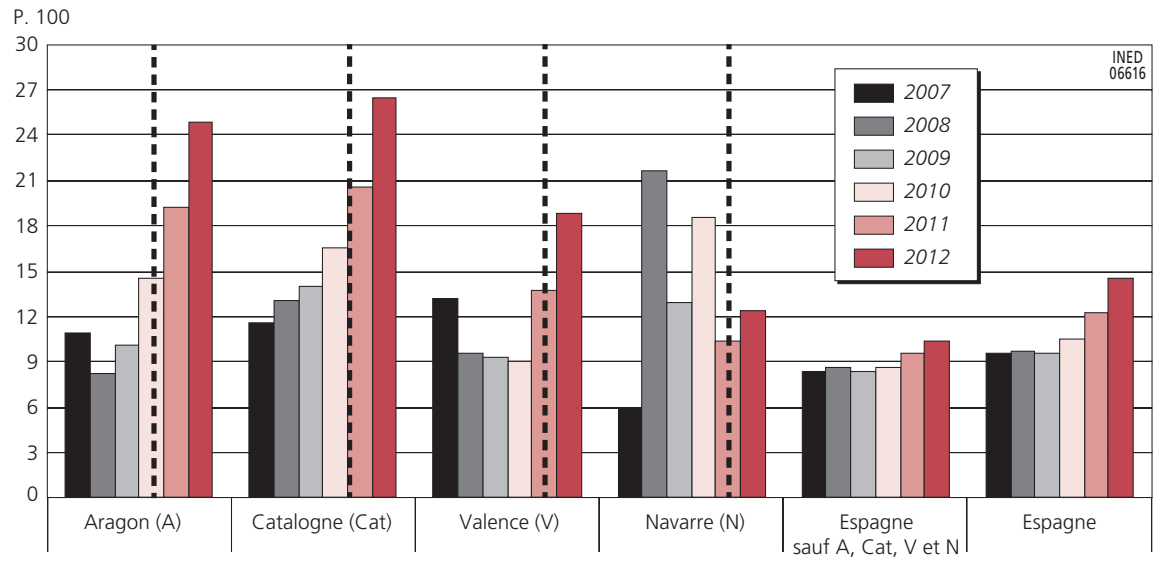

Note: Comme le montrent les barres verticales, les lois sur la famille ont été votées le 26 aout 2010 en Aragon, le $1^{\text {er }}$ janvier 2011 en Catalogne, le 25 mai 2011 dans la région de Valence et le 28 juin en Navarre.

Source : www.ine.es

La Catalogne a été choisie pour effectuer des comparaisons avec les autres régions à cause de la spécificité reconnue de son Code civil qui favorise une parentalité partagée plutôt qu'une simple garde partagée. Cette région représente un sixième de la population espagnole, et la tendance y est clairement l'augmentation constante des gardes partagées depuis 2007 (par comparaison à la courbe en U à Valence et aux niveaux de la Navarre plus élevés avant le nouveau Code civil que dans les années suivantes).

Historiquement, la Catalogne est souvent perçue comme ayant joué un rôle de précurseur en termes de comportements démographiques. Les déclins successifs de la mortalité et de la fécondité s'y sont produits avant la plupart des autres régions espagnoles (depuis la première transition démographique). Des tendances similaires peuvent être observées pour d'autres phénomènes sociodémographiques associés à ce qu'on appelle la seconde transition démographique, notamment l'âge au mariage, la proportion de naissances hors mariage et le taux de divorce. Tous ces phénomènes ont été plus précoces ou plus intenses en Catalogne que dans l'ensemble de l'Espagne (Solsona, 1997). Il existe en outre une nette différence entre la Catalogne et le reste de l'Espagne en matière de développement social et économique au cours du dernier siècle. En termes macroéconomiques, la Catalogne a toujours été plus riche et, par exemple, la participation des mères catalanes en couple à la population active est également plus élevée (66 \% contre 57 \% pour le reste de l'Espagne selon le recensement de 2001). Ces éléments affectent les comportements démographiques et sont susceptibles d'expliquer en partie les différences avec le reste de l'Espagne (Cabré Pla, 1999; Simó et al., 2000; Spijker et Blanes-Llorens, 2009). 


\section{Le Code civil catalan de 2010}

Le livre II du CCC portant sur la personne et la famille a été approuvé le 29 juillet 2010 et est entré en vigueur le $1^{\text {er }}$ janvier 2011. Il traite, entre autres aspects $^{(8)}$, des conséquences de la rupture du mariage pour les enfants mineurs.

La partie de la réforme espagnole sur le divorce de 2005 traitant de la garde partagée légale met en avant le concept d'autorité parentale, tandis que le CCC insiste sur la notion de responsabilité parentale (article 233-8). Par ailleurs, le terme de garde partagée n'est pas spécifiquement mentionné dans le CCC, qui fait plutôt allusion aux statuts de parent gardien et aux conditions d'établissements des accords et jugements liées à la rupture de toute relation qui implique des décisions concernant la charge d'enfants mineurs. Il est également rarement fait mention de garde exclusive. Il est important de noter que la loi catalane a donc tendance à considérer que l'accord mutuel entre les parties et toutes les décisions judiciaires liées doivent être cohérents avec les conditions de vie affectives et matérielles des deux anciens partenaires et du ou des enfants qu'ils ont en commun.

Le nouveau CCC a pour objectif principal d'encourager les différentes formes de coparentalité et de médiation. Il introduit deux outils puissants pour reconnaître et encourager la parentalité partagée : le juge doit, d'une part, décrire la situation et les accords entre parties (article 233-11) et, d'autre part, prévoir le mode de garde de l'enfant (article 233-9).

Le juge doit s'appuyer sur des critères clairement établis, tenir compte des circonstances particulières de chaque situation et déterminer le régime et les modalités de la garde des enfants selon les critères suivants :

- La relation spécifique de l'enfant avec ses parents.

- Le dévouement du père ou de la mère vis-à-vis des enfants avant la rupture.

- La capacité des parents à garantir le bien-être des enfants et un environnement de vie adapté.

- L'attitude de chaque parent vis-à-vis d'une coopération mutuelle assurant un maximum de stabilité dans la relation de l'enfant avec les deux parents.

- Lopinion de l'enfant.

- Les accords passés préalablement à la dissolution de l'union.

- La localisation du foyer de chaque parent et les emplois du temps et activités des enfants et des parents.

Le CCC établit également que les propositions des parents après une rupture familiale doivent être incluses dans les procédures judiciaires. Cela prend

(8) Le livre II du CCC portant sur la personne et la famille (Loi 25/2010) est divisé en 4 sections : I) Les personnes physiques, II) Les institutions chargées de la protection des personnes, III) La famille, IV) Les autres relations de coexistence (parentales, couples stables et belles-familles) et d'aide mutuelle, http://civil.udg.es/normacivil/cat/ccc/Index.htm (en catalan)

http://www.boe.es/boe/dias/2010/08/21/pdfs/BOE-A-2010-13312.pdf (en espagnol). 
la forme d'un plan parental qui détaille les engagements parentaux sur la garde de l'enfant, les soins qu'il convient de lui prodiguer et la coopération entre la mère et le père après le divorce. Concrètement, le CCC établit que le plan parental doit comprendre des accords sur les points suivants :

- Le ou les lieux où l'enfant vit habituellement. Cela doit comprendre des règles destinées à déterminer à tout moment quel parent est le gardien responsable, ce qui inclut également la gestion des activités quotidiennes.

- Laccord sur les séjours des enfants chez chaque parent, notamment pendant les périodes de vacances et autres dates importantes pour les enfants, les parents et la famille et, le cas échéant, comment les coûts sont partagés.

- Les moyens pour communiquer et entretenir une relation durant les périodes où l'une ou l'autre parent n'est pas présent.

- Le type d'éducation (et les activités extra-scolaires), les activités sportives et de loisirs lorsque cela est approprié.

- L'obligation de partager l'information sur l'éducation, la santé et le bienêtre de l'enfant.

En cas de conflits ou d'évolution des besoins de l'enfant, il est possible d'avoir recours à une médiation familiale pour résoudre ces conflits ou de modifier le plan en l'adaptant aux nouveaux besoins de l'enfant aux différentes étapes de sa vie. Si le divorce s'effectue sur la base d'un consentement mutuel, le juge accepte habituellement le plan parental qui est inclus dans la décision. S'il n'y a pas d'accord ou si le plan parental n'est pas approuvé, les conditions de résidence des enfants sont déterminées par le tribunal. En tenant compte des critères mentionnés ci-dessus, le juge décide comment les responsabilités parentales sont exercées et, en particulier, qui obtient le droit de résidence de l'enfant. Cette décision est basée sur les caractéristiques des parents et, pardessus tout, les intérêts de l'enfant.

Le plan parental n'impose aucun type spécifique d'organisation. Même si le jugement de divorce est contesté, il encourage les parents à s'organiser entre eux pour répondre aux besoins de leur enfant, anticiper ses problèmes (ainsi que les solutions possibles) et prendre les décisions qui affecteront sa vie. De plus, les décisions sont prises en fonction des capacités économiques des parents, sans relation directe avec l'attribution de la garde. Le plan parental facilite également la collaboration entre les avocats des deux parties et les psychologues, psychiatres, éducateurs et assistants sociaux indépendants, chacun de ces acteurs intervenant sur des aspects spécifiques liés à la rupture avant que les parents n'entament les procédures de divorce. Cela facilite l'établissement d'accords, la transparence et le respect des engagements pris. Lautre nouveauté du plan parental consiste à affirmer que l'échec de la vie conjugale des parents ne signifie pas automatiquement que les enfants sont séparés d'un parent et confiés à un autre. 
Les objectifs de la réforme espagnole sur le divorce de 2005 et du Code civil catalan de 2010 étaient de renforcer la coresponsabilité des parents en cas de rupture de leur relation et d'encourager la médiation en cas de conflits. Cependant, non seulement le CCC établit explicitement un critère spécifique pour décider de la garde des enfants, mais il introduit également dans les procédures judiciaires un plan parental qui renforce la capacité des parents à s'organiser entre eux pour s'occuper de leurs enfants. Le juge détermine la prestation compensatoire ainsi que l'utilisation du logement familial jusqu'à ce que le plus jeune enfant atteigne l'âge de 18 ans. Ces décisions sont prises en fonction de la capacité économique des parents et ne sont donc pas nécessairement liées à l'attribution de la garde.

Tandis que la Catalogne affichait déjà une plus grande proportion de gardes partagées que le reste de l'Espagne avant le CCC de 2010, nous supposons que ces différences se sont creusées depuis, en particulier après avoir pris en compte les caractéristiques individuelles des mariages et des divorces susceptibles d'être liées aux attributions de garde partagée en Espagne et en Catalogne (Spijker, 2012; Solsona et al., 2014).

\section{Données et méthodes}

Les données utilisées dans cette étude proviennent de l'Institut national de la statistique espagnol (INE). Conformément à un accord passé entre l'INE et le Conseil général du pouvoir judiciaire, le 14 février 1995, l'INE a publié pour la première fois des statistiques judiciaires (estadísticas judiciales) sur les séparations d'unions mariées, les divorces et annulations fournies par les tribunaux ${ }^{(9)}$. La réforme sur le divorce de 2005 a accéléré les procédures judiciaires et amélioré l'enregistrement statistique de ces jugements ${ }^{(10)}$. À des fins de recherche, des informations anonymisées portant sur environ deux tiers des jugements sont disponibles sur demande sous la forme de données individuelles (66988 jugements en 2012) ${ }^{(11)}$. Elles sont transmises à la demande de l'INE. Depuis 2007, elles comprennent des données sur les accords de garde et les dissolutions de mariages homosexuels (les deux ayant

(9) Les tribunaux remplissent un bulletin statistique pour chaque jugement prononcé, appelé boletines estadísticos de sentencias de separación, divorcio y nulidad.

(10) Depuis 2007, cet enregistrement s'effectue directement sur une plateforme virtuelle baptisée Punto Neutro Judicial.

(11) La majorité des couples mariés avec enfants mineurs qui décident de divorcer ou de se séparer le font de manière formelle par l'intermédiaire d'une procédure judiciaire, bien que cela ne soit pas une obligation. C'est le point de vue des avocats consultés. La part des propriétaires de logements étant très importante en Espagne, le divorce permet de régler simultanément la prise en charge des enfants et la division de la propriété. Nous ne disposons cependant pas d'autres études ou données pour le confirmer. 
été juridiquement formalisés en 2005), mais elles ne comprennent pas d'information sur les couples non mariés qui se sont séparés ${ }^{(12)}$.

Pour cette étude, nous avons analysé les données des jugements prononcés sur la période 2007-2012, mais uniquement pour les couples hétérosexuels officiellement mariés et juridiquement divorcés ${ }^{(13)}$. Chaque registre de divorce contient des détails démographiques sur chacun des époux et sur le mariage, ainsi que des informations sur la procédure judiciaire. Les données individuelles précisent la date de naissance, le sexe, le statut marital avant le mariage étudié et la nationalité de chacun des époux. Les données comprennent également les dates du mariage, de la demande et du jugement de dissolution du mariage. Nous avons donc pu calculer les âges au mariage et au divorce, la durée du mariage (jusqu'au début de la procédure de divorce et jusqu'au jugement) et la durée de la procédure de divorce elle-même. Les informations sur la procédure judiciaire précisent quel époux demande la dissolution du mariage (ou les deux), le contenu du jugement (divorce, séparation ou annulation), l'existence d'une séparation antérieure, la contestation éventuelle de la procédure judiciaire, la personne qui doit payer une pension alimentaire ou une prestation compensatoire, le nombre de mineurs impliqués et - particulièrement intéressant pour notre étude- le type de résidence des enfants. Cependant, il n'y a aucune information sur l'âge des enfants, le niveau d"éducation ou le niveau de revenu des parents.

Concernant la méthode d'analyse, nous allons, dans un premier temps, montrer l'évolution de la fréquence de chaque mode de garde entre 2007 et 2012, en comparant la Catalogne avec le reste de l'Espagne, puis décrire plus en détail la prévalence de la garde partagée pour les deux territoires en fonction des variables mentionnées ci-dessus. Nous avons regroupé les années en deux périodes : 2007-2010 et 2011-2012, à savoir les périodes précédant et suivant l'introduction du CCC. Enfin, nous avons réalisé une régression logistique multivariée avec ces mêmes variables (en excluant certaines variables trop fortement corrélées à d'autres), ce qui nous permet de vérifier l'effet propre de chaque facteur sur la propension à accorder la garde partagée (par rapport au fait de ne pas l'accorder) en Catalogne et dans le reste de l'Espagne. Dans ce cas, les données ne sont plus regroupées par année.

(12) Les décisions sur la procédures de séparation sont disponibles sur le site CGPJ (www.poderjudicial.es/). Cependant, même si elles ne comprennent pas de détails sur les caractéristiques des individus, des mariages ou des accords de garde établis, les données individuelles indiquent le total des dissolutions pour les couples non mariés avec des enfants mineurs. En 2012, environ 33 \% des dissolutions d'union impliquant des enfants mineurs concernaient des couples non mariés (contre $18 \%$ en 2007).

(13) Léchantillon, par conséquent, exclut également les couples mariés juridiquement séparés, mais non divorcés (10\% du nombre total des dissolutions de mariage en Espagne en 2007, 5 \% en 2012). La loi exigeait la séparation de corps avant le divorce jusqu'en 2005, et cela est encore possible aujourd'hui; par conséquent, les chiffres de la séparation et du divorce ne peuvent être analysés ensemble, car les dissolutions de mariage en deux étapes seraient comptabilisées deux fois si elles avaient lieu durant la même période d'étude. C'est pourquoi nous excluons les séparations. 


\section{Résultats et discussion}

En 2007, la première année où les données concernant les accords sur la résidence des enfants ont été enregistrées, $11,6 \%$ des jugements de divorce prononcés en Catalogne prévoyaient une garde partagée, contre 9,2\% pour le reste de l'Espagne. Cette différence de seulement 2,4 points suggère que la réforme sur le divorce de 2005 a eu un effet immédiat dans l'ensemble du pays. Jusqu'en 2010, l'année où le CCC a été mis en œuvre, la proportion reste stable dans toute l'Espagne, alors qu'elle augmente de manière relative et absolue en Catalogne et représente 16,6\% de tous les modes de garde. Même si deux ans plus tard la proportion de gardes alternées a progressé d'un tiers dans le reste de l'Espagne, atteignant $12 \%$, elle était plus de deux fois supérieure en Catalogne (26,4\%). Cette différence accrue pourrait être une conséquence du nouveau CCC (tableau 1).

Étant donné les différences territoriales manifestes en matière d'attribution de garde partagée, nous pouvons maintenant déterminer si des caractéristiques issues des données sur les jugements renforcent ou réduisent la probabilité de décision d'une garde partagée. Le tableau 2 montre la probabilité d'obtenir une garde partagée en fonction des caractéristiques des individus, des couples, des divorces et des procédures judiciaires pour la période précédent le CCC (20072010) et pour la période suivante (2011-2012). Les résultats sont fournis pour la Catalogne et le reste de l'Espagne, et ils sont synthétisés ci-dessous en trois points principaux.

Les premiers résultats concernent la fréquence de la garde partagée. Dans la période 2007-2010, la proportion de gardes partagées était en moyenne 1,5 fois supérieure en Catalogne par rapport au reste de l'Espagne. Ce ratio progresse d'environ 0,2 point par an, une tendance qui s'est poursuivie en 2011-2012, lorsque le ratio a atteint 2,1 (23,8 \% des accords de garde étaient conjoints en Catalogne contre 11,3\% pour le reste de l'Espagne).

Deuxièmement, nous avons analysé les caractéristiques des époux, des mariages et des procédures judiciaires. Malgré les différences de proportions de gardes partagées entre la Catalogne et le reste de l'Espagne, les deux territoires présentent quasiment les mêmes variations de la fréquence de ce mode de garde selon les caractéristiques personnelles. Par exemple, les pères âgés de 30 à 34 ans et les mères âgées de 25 à 29 ans au moment du mariage ont le plus de chances d'aboutir à une garde conjointe (cela s'applique aux deux périodes). En outre, le ratio entre la Catalogne et le reste de l'Espagne est identique pour la plupart des catégories de divorces, en particulier pour la période 2007-2010. Lorsqu'on examine les variables associées aux caractéristiques du mariage, la majorité des catégories affichent peu de différences en termes de proportion de gardes physiques conjointes lorsqu'on les compare à la moyenne ou au ratio entre la Catalogne et le reste de l'Espagne. Il existe toutefois une exception : lorsque l'un ou les deux époux sont étrangers, les différences territoriales sont relativement minces : 
Tableau 1. Accords sur le mode de résidence des enfants pour les parents qui divorcent en Catalogne et dans le reste de l'Espagne, 2007-2012

\begin{tabular}{|c|c|c|c|c|c|c|c|c|}
\hline \multirow{2}{*}{ Garde (\%) } & \multicolumn{6}{|c|}{ Année } & \multicolumn{2}{|c|}{ Variations relatives (\%) } \\
\hline & 2007 & 2008 & 2009 & 2010 & 2011 & 2012 & $2007-2010$ & 2010-2012 \\
\hline \multicolumn{9}{|c|}{ Catalogne } \\
\hline Mère & 83,5 & 82,1 & 79,4 & 77,8 & 73,1 & 67,6 & $-6,8$ & $-13,1$ \\
\hline Père & 5,0 & 4,5 & 6,0 & 4,9 & 5,4 & 5,1 & $-1,8$ & $+3,8$ \\
\hline Partagée & 11,6 & 13,1 & 14,0 & 16,6 & 20,6 & 26,4 & $+43,3$ & $+59,7$ \\
\hline Autre & 0,0 & 0,3 & 0,6 & 0,8 & 1,0 & 0,9 & & $+21,5$ \\
\hline Ensemble & 100,0 & 100,0 & 100,0 & 100,0 & 100,0 & 100,0 & & \\
\hline Effectif total & 11967 & 10540 & 9533 & 10242 & 9775 & 9962 & $-14,4$ & $-2,7$ \\
\hline $\begin{array}{l}\text { Dont garde } \\
\text { partagée }\end{array}$ & 1383 & 1384 & 1333 & 1696 & 2014 & 2634 & $+22,6$ & $+55,3$ \\
\hline \multicolumn{9}{|c|}{ Espagne hors Catalogne } \\
\hline Mère & 86,1 & 86,7 & 85,1 & 84,5 & 83,5 & 82,1 & $-1,9$ & $-2,8$ \\
\hline Père & 4,8 & 4,0 & 5,5 & 5,8 & 5,4 & 5,4 & $+22,8$ & $-7,6$ \\
\hline Partagée & 9,2 & 8,9 & 8,7 & 9,1 & 10,4 & 12,0 & $-1,3$ & $+32,5$ \\
\hline Autre & 0,1 & 0,3 & 0,8 & 0,6 & 0,7 & 0,5 & & $-21,9$ \\
\hline Ensemble & 100,0 & 100,0 & 100,0 & 100,0 & 100,0 & 100,0 & & \\
\hline Effectif total & 53025 & 48598 & 42856 & 44169 & 45202 & 45380 & $-16,7$ & $+2,7$ \\
\hline $\begin{array}{l}\text { Dont garde } \\
\text { partagée }\end{array}$ & 4866 & 4332 & 3713 & 3999 & 4715 & 5445 & $-17,8$ & $+36,2$ \\
\hline \multicolumn{9}{|c|}{$\begin{array}{l}\text { Note : La catégorie «Autre » regroupe les cas très rares où la garde est attribuée par les tribunaux à une tierce } \\
\text { partie (un grand-parent, un autre parent ou, si ce n'est pas possible, une institution). Les changements relatifs } \\
\text { ne sont pas calculés pour cette catégorie en raison de la faiblesse des effectifs. } \\
\text { Champ : Parents qui divorcent avec enfant mineur de } 2007 \text { à } 2012 \text {. } \\
\text { Source : www.ine.es }\end{array}$} \\
\hline
\end{tabular}

10,6 \% de garde conjointe en Catalogne en 2011-2012 quand les deux conjoints sont étrangers contre $8,1 \%$ dans le reste du pays, ces chiffres sont respectivement de 25,3\% et 11,6 \% lorsque les deux époux sont Espagnols. Dans les deux territoires, les parents divorcés avec deux enfants sont plus enclins à obtenir une garde partagée que lorsqu'il y a un ou trois enfants ou plus. Bien que les résultats issus d'autres recherches sur l'effet du nombre d'enfants soient mitigés, des résultats similaires ont été obtenus par Juby et al. (2005) et Turunen (2015). Aucune des deux études ne fournit d'explication, mais nous pouvons cependant évoquer plusieurs raisons possibles. Tout d'abord, de nombreuses mères ne sont pas prêtes à renoncer à la garde exclusive lors d'un divorce (Seltzer, 1994). Il serait intéressant de voir si elles sont plus disposées à le faire dans le cas des couples avec enfant unique. Par ailleurs, les grandes familles ont souvent une division traditionnelle du travail, et la plupart des couples qui se séparent n'ont pas les moyens financiers d'avoir deux logements assez grands pour accueillir une famille nombreuse (Juby et al., 2005), ce qui réduit la probabilité de demande de garde partagée.

Concernant la probabilité d'attribution de garde partagée en fonction du type de procédure judiciaire, elle semble plus élevée lorsque les deux époux 
Tableau 2. Pourcentage de parents divorcés ayant obtenu une garde partagée en fonction des caractéristiques du couple, de l'union et de la procédure judiciaire, 2007-2010 et 2011-2012,

Catalogne et reste de l'Espagne

\begin{tabular}{|c|c|c|c|c|c|c|}
\hline \multirow[t]{2}{*}{ Variable / Catégories } & \multicolumn{2}{|c|}{ 1. Catalogne } & \multicolumn{2}{|c|}{$\begin{array}{l}\text { 2. Espagne (hors } \\
\text { Catalogne) }\end{array}$} & \multicolumn{2}{|c|}{ Ratio $1 / 2$} \\
\hline & $2007-2010$ & 2011-2012 & $2007-2010$ & 2011-2012 & $2007-2010$ & 2011-2012 \\
\hline Année de divorce & & & & & & \\
\hline 2007 & 11,5 & & 9,2 & & 1,3 & \\
\hline 2008 & 13,2 & & 8,9 & & 1,5 & \\
\hline 2009 & 14,1 & & 8,7 & & 1,6 & \\
\hline 2010 & 16,7 & & 9,1 & & 1,8 & \\
\hline 2011 & & 20,8 & & 10,5 & & 2,0 \\
\hline 2012 & & 26,7 & & 12,1 & & 2,2 \\
\hline
\end{tabular}

\begin{tabular}{|c|c|c|c|c|c|c|}
\hline \multicolumn{7}{|c|}{ Caractéristiques des conjoints mariés } \\
\hline \multicolumn{7}{|c|}{ Âge du père au mariage } \\
\hline$<25$ ans & 12,8 & 19,7 & 8,4 & 9,6 & 1,5 & 2,1 \\
\hline $25-29$ ans & 14,1 & 25,1 & 9,3 & 11,6 & 1,5 & 2,2 \\
\hline 30-34 ans & 14,4 & 26,3 & 9,4 & 12,3 & 1,5 & 2,1 \\
\hline 35 ans et + & 14,0 & 22,8 & 9,1 & 12,1 & 1,5 & 1,9 \\
\hline \multicolumn{7}{|c|}{ Âge de la mère au mariage } \\
\hline$<25$ ans & 13,3 & 22,0 & 8,8 & 10,2 & 1,5 & 2,2 \\
\hline $25-29$ ans & 14,6 & 25,6 & 9,5 & 12,5 & 1,5 & 2,0 \\
\hline $30-34$ ans & 13,7 & 25,1 & 8,8 & 11,5 & 1,5 & 2,2 \\
\hline 35 ans et + & 12,7 & 22,3 & 8,5 & 11,3 & 1,5 & 2,0 \\
\hline \multicolumn{7}{|c|}{ Âge du père au divorce } \\
\hline$<30$ ans & 10,7 & 15,7 & 7,2 & 9,1 & 1,5 & 1,7 \\
\hline $30-34$ ans & 12,4 & 21,3 & 8,1 & 9,6 & 1,5 & 2,2 \\
\hline $35-39$ ans & 13,9 & 24,8 & 8,7 & 10,8 & 1,6 & 2,3 \\
\hline $40-44$ ans & 14,2 & 26,1 & 9,4 & 12,1 & 1,5 & 2,2 \\
\hline $45-49$ ans & 14,4 & 24,2 & 9,6 & 11,8 & 1,5 & 2,0 \\
\hline 50 ans et + & 13,6 & 20,1 & 9,4 & 11,7 & 1,4 & 1,7 \\
\hline \multicolumn{7}{|c|}{ Âge de la mère au divorce } \\
\hline$<30$ ans & 11,2 & 19,0 & 7,6 & 9,5 & 1,5 & 2,0 \\
\hline $30-34$ ans & 13,2 & 22,8 & 8,4 & 10,2 & 1,6 & 2,2 \\
\hline $35-39$ ans & 14,1 & 25,6 & 9,1 & 11,4 & 1,5 & 2,2 \\
\hline $40-44$ ans & 14,2 & 24,2 & 9,3 & 12,0 & 1,5 & 2,0 \\
\hline $45-49$ ans & 14,1 & 23,7 & 9,5 & 12,1 & 1,5 & 2,0 \\
\hline 50 ans et + & 13,8 & 20,5 & 9,6 & 10,7 & 1,4 & 1,9 \\
\hline \multicolumn{7}{|c|}{ Situation précédente du père } \\
\hline Célibataire & 13,9 & 23,9 & 9,0 & 11,3 & 1,5 & 2,1 \\
\hline Divorcé / veuf & 12,2 & 21,2 & 8,6 & 10,4 & 1,4 & 2,0 \\
\hline \multicolumn{7}{|c|}{ Situation précédente de la mère } \\
\hline Célibataire & 13,9 & 23,9 & 9,0 & 11,3 & 1,6 & 2,1 \\
\hline Divorcée / veuve & 11,8 & 22,0 & 9,7 & 10,0 & 1,2 & 2,2 \\
\hline \multicolumn{7}{|c|}{ Caractéristiques du mariage } \\
\hline \multicolumn{7}{|c|}{ Différence d'âge des mariés } \\
\hline Même âge & 14,4 & 24,0 & 9,1 & 11,6 & 1,6 & 2,1 \\
\hline Âge $\mathrm{F}<$ âge $\mathrm{H}$ & 12,7 & 23,5 & 8,2 & 10,4 & 1,5 & 2,3 \\
\hline Âge $\mathrm{F}>$ âge $\mathrm{H}$ & 13,4 & 23,7 & 9,1 & 11,3 & 1,5 & 2,1 \\
\hline
\end{tabular}


Tableau 2 (suite). Pourcentage de parents divorcés ayant obtenu une garde partagée en fonction des caractéristiques du couple, de l'union et de la procédure judiciaire, 2007-2010 et 2011-2012, Catalogne et reste de l'Espagne

\begin{tabular}{|c|c|c|c|c|c|c|}
\hline \multirow[t]{2}{*}{ Variable / Catégories } & \multicolumn{2}{|c|}{ 1. Catalogne } & \multicolumn{2}{|c|}{$\begin{array}{l}\text { 2. Espagne (hors } \\
\text { Catalogne) }\end{array}$} & \multicolumn{2}{|c|}{ Ratio $1 / 2$} \\
\hline & $2007-2010$ & $2011-2012$ & $2007-2010$ & $2011-2012$ & $2007-2010$ & $2011-2012$ \\
\hline \multicolumn{7}{|l|}{ Nationalité des mariés } \\
\hline 2 Espagnols & 14,2 & 25,3 & 9,0 & 11,6 & 1,6 & 2,2 \\
\hline 1 Espagnol et 1 étranger & 11,0 & 18,8 & 9,0 & 10,4 & 1,2 & 1,8 \\
\hline 2 étrangers & 9,3 & 10,6 & 7,6 & 8,1 & 1,2 & 1,3 \\
\hline \multicolumn{7}{|l|}{ Durée du mariage } \\
\hline$<5$ ans & 12,0 & 20,8 & 7,7 & 9,5 & 1,6 & 2,2 \\
\hline $5-10$ ans & 14,4 & 24,4 & 8,5 & 11,3 & 1,7 & 2,1 \\
\hline $10-20$ ans & 13,7 & 25,1 & 9,6 & 12,0 & 1,4 & 2,1 \\
\hline 20 ans et + & 14,0 & 21,2 & 8,9 & 10,5 & 1,6 & 2,0 \\
\hline \multicolumn{7}{|l|}{ Nombre d'enfants mineurs } \\
\hline 1 & 12,9 & 21,8 & 8,5 & 10,7 & 1,5 & 2,0 \\
\hline 2 & 15,2 & 26,8 & 9,8 & 12,2 & 1,5 & 2,2 \\
\hline 3 et + & 13,8 & 20,9 & 9,0 & 10,9 & 1,5 & 1,9 \\
\hline
\end{tabular}

\begin{tabular}{|c|c|c|c|c|c|c|}
\hline \multicolumn{7}{|c|}{ Procédure judiciaire } \\
\hline \multicolumn{7}{|l|}{ Requérant } \\
\hline Le mari & 13,0 & 23,3 & 8,7 & 12,0 & 1,5 & 1,9 \\
\hline La femme & 9,4 & 15,8 & 6,4 & 6,6 & 1,5 & 2,4 \\
\hline Les deux & 17,2 & 29,5 & 11,4 & 14,5 & 1,5 & 2,0 \\
\hline \multicolumn{7}{|l|}{ Séparation préalable } \\
\hline Oui & 10,5 & 16,8 & 7,0 & 7,9 & 1,5 & 2,1 \\
\hline Non & 14,5 & 24,5 & 9,5 & 11,6 & 1,5 & 2,1 \\
\hline \multicolumn{7}{|l|}{ Durée de la procédure } \\
\hline$<6$ mois & 15,2 & 27,7 & 10,1 & 13,1 & 1,5 & 2,1 \\
\hline 6-11 mois & 11,0 & 17,9 & 7,2 & 8,2 & 1,5 & 2,2 \\
\hline 12 mois et + & 9,9 & 12,9 & 6,6 & 7,0 & 1,5 & 1,8 \\
\hline \multicolumn{7}{|l|}{ Jugement } \\
\hline Consentement mutuel & 15,8 & 27,8 & 10,9 & 14,0 & 1,4 & 2,0 \\
\hline Divorce contesté & 8,5 & 13,3 & 6,0 & 6,6 & 1,4 & 2,0 \\
\hline \multicolumn{7}{|l|}{ Prestation compensatoire } \\
\hline Payée par le mari & 16,7 & 27,2 & 10,9 & 10,3 & 1,5 & 2,6 \\
\hline Payée par la femme & 20,0 & 31,6 & 18,2 & 11,2 & 1,1 & 2,8 \\
\hline Payée par les deux & 13,4 & 23,3 & 8,7 & 11,4 & 1,5 & 2,0 \\
\hline \multicolumn{7}{|l|}{ Pension alimentaire } \\
\hline Payée par le mari & 7,6 & 10,1 & 5,5 & 4,7 & 1,4 & 2,1 \\
\hline Payée par la femme & 8,0 & 11,5 & 6,5 & 8,5 & 1,2 & 1,3 \\
\hline Payée par les deux & 83,7 & 90,2 & 73,7 & 87,1 & 1,1 & 1,0 \\
\hline Total & 13,8 & 23,8 & 9,0 & 11,3 & 1,5 & 2,1 \\
\hline Effectif (non pondéré) & 28246 & 12435 & 124357 & 55216 & & \\
\hline \multicolumn{7}{|c|}{$\begin{array}{l}\text { Note : Fréquences pondérées (sur le nombre annuel de divorces) et effectifs non pondérés. La catégorie affichant } \\
\text { la plus forte proportion et le rapport de cotes (odds ratio) le plus élevé entre la Catalogne et le reste de l'Espagne } \\
\text { est indiquée en gras. } \\
\text { Champ : Parents qui divorcent avec enfant mineur de } 2007 \text { à } 2012 \text {. } \\
\text { Source : Calculs des auteurs basés sur les données individuelles des jugements de divorce obtenues auprès de } \\
\text { I'INE. }\end{array}$} \\
\hline
\end{tabular}


sont requérants (les deux ayant probablement un accord a priori sur cette question), lorsqu'il n'y a pas de séparation préalable, lorsque la procédure de divorce est rapide et, bien sûr, si le divorce se fait sur la base du consentement mutuel. Rappelons que le ratio de garde partagée entre la Catalogne et l'Espagne reste approximativement de 1,5 durant la période 2007-2010 et proche ou égal de la moyenne de 2,1 en 2011-2012 pour la plupart des catégories ${ }^{(14)}$. Parmi les procédures plus longues (12 mois et plus), la progression de la garde conjointe en Catalogne a été plus modeste et la différence avec le reste du pays est plus faible en 2011-2012. Les deux dernières variables de la procédure judiciaire portent sur les pensions versées aux conjoints et aux enfants. Cette décision est prise après la décision du juge sur la garde. Dans la plupart des cas de garde partagée (90\%), les deux époux payent une pension alimentaire (montant calculé sur les besoins de base de l'enfant : nourriture, vêtements, frais scolaires, de santé...). La garde partagée en Catalogne est plus importante lorsque la femme paye la pension. Ces deux résultats suggèrent que ce type de garde est privilégié par les familles non traditionnelles où la femme est susceptible de travailler. Même s'il s'agit d'une simple hypothèse (dans la mesure où nous ne disposons d'aucune information sur l'emploi ou le revenu), elle est plausible dans la mesure où les femmes ont plus de pouvoir de négociation sur la répartition des soins aux enfants avec le père si elles disposent d'une autonomie économique et si elles contribuent au paiement de la pension. Cela est directement lié à l'entrée des femmes mariées sur le marché du travail, un phénomène plus prégnant en Catalogne que dans le reste de l'Espagne depuis de nombreuses décennies (Solsona, 1997).

Troisièmement, une analyse multivariée a été menée pour vérifier l'effet indépendant de chaque caractéristique ${ }^{(15)}$ sur l'attribution de la garde partagée. Pour vérifier l'influence du CCC sur la prévalence des gardes partagées en Catalogne, la Catalogne et le reste de l'Espagne ont été analysés séparément. Cela permet également de comparer directement les rapports de cotes des variables et donc de vérifier si certaines caractéristiques spécifiques contribuent à l'octroi plus important de la garde partagée en Catalogne. Les résultats montrent qu'en dépit d'un niveau constamment plus élevé de garde conjointe en Catalogne, l'effet de la plupart des caractéristiques sur l'occurrence de ce mode de garde n'est pas très différent du reste de l'Espagne. Comme on peut le voir dans l'analyse bivariée, la principale exception est la nationalité des époux : en Catalogne, les couples mixtes ou étrangers sont moins susceptibles d'obtenir la garde partagée que les couples autochtones; tandis que dans le reste de l'Espagne, les couples étrangers n'ont qu'une chance légèrement plus

(14) Sauf à l'exception notable des cas où la femme est requérante.

(15) La variable de l'âge de la femme au divorce était fortement corrélée à l'âge du mari au divorce, et n'a par conséquent pas été incluse dans l'analyse multivariée. Lầge au mariage pour les deux types de couples a également été exclu, car il peut être dérivé des variables de l'âge au divorce et de la durée du mariage. Ni la prestation compensatoire ni la pension alimentaire n'ont été testées, car c'est plutôt l'attribution de la garde qui détermine qui paye que l'inverse. 
faible d'avoir ce mode de garde. Cela s'explique probablement par la plus forte présence en Catalogne de migrants issus de régions du monde où les rôles de genre sont plus traditionnels (en particulier l'Amérique latine et l'Afrique du Nord) par rapport aux migrants de pays plus égalitaires (la plupart des pays européens).

Une autre exception doit être relevée : les mariages ayant duré entre 10 et 20 ans ont également une plus forte propension à obtenir la garde partagée dans le reste de l'Espagne, alors que ce n'est pas le cas en Catalogne. Cependant, le principal résultat de l'analyse indique que le CCC a un effet supplémentaire au-delà de la différence territoriale existante. Comme on peut le voir dans le tableau 3 et la figure 3, nous observons qu'après avoir pris en compte les caractéristiques du jugement, les chances d'attribution de garde partagée augmentent en Catalogne entre 2007 (l'année de référence) et 2010 de $44 \%$ (de 1,00 à 1,44), mais elles reculent dans le reste de l'Espagne de $7 \%$ (de 1,00 à 0,93). Cependant, cet écart se creuse encore entre 2010 et 2012 avec la progression du rapport des chances de $89 \%$ (de 1,44 à 2,72) en Catalogne contre $35 \%$ (de 0,93 à 1,26) dans le reste de l'Espagne ${ }^{(16)}$

Comme nous pouvons le voir sur les figures 2 et 3 et le tableau 1, la garde partagée est plus de deux fois plus souvent présente en Catalogne que dans le reste de l'Espagne. La partie du CCC liée à la garde des enfants n'a fait qu'accentuer cette différence. Lorsqu'on effectue la comparaison avec les communautés autonomes d'Espagne qui ne disposent pas de loi sur la famille, le CCC comprend une prescription particulièrement importante favorisant la garde partagée -à savoir que le plan parental doit détailler les engagements pris par chaque parent divorcé par rapport à la garde, les soins et l'éducation de l'enfant.

S'il peut être surprenant au premier abord que le terme de " garde partagée " ne soit pas spécifiquement défini dans le CCC, il existe des articles qui facilitent l'attribution (ou la non-attribution) de la garde partagée, en particulier le plan parental et les critères et circonstances utilisés par le juge pour déterminer le type de garde tels que nous les avons évoqués dans la section II.

La probabilité que la garde partagée soit attribuée est plus élevée si les deux parents souhaitent partager la garde et si l'évaluation par le juge des circonstances mentionnées ci-dessus est positive. Le premier point peut être illustré à l'aide des données sur les jugements. Par exemple, nos résultats montrent qu'en 20112012, la garde conjointe a été attribuée en Catalogne pour 27,8 \% des divorces avec consentement mutuel contre 15,8 \% lorsque les divorces sont contestés. Cela est encore plus manifeste lorsque les données sont analysées de manière inverse, la proportion de jugements de divorces par consentement mutuel étant

(16) Nous avons également testé un modèle pour toute l'Espagne, où nous avons ajouté une variable indicatrice pour la région ( 1 = Catalogne, 0 = reste de l'Espagne) et testé les interactions entre la région et l'époque. Les résultats (qui peuvent être obtenus auprès des auteurs) sont très similaires, car ils montrent que la tendance s'est accélérée après 2010, tandis la garde partagée progressait déjà en Catalogne avant l'application de la nouvelle législation le $1^{\mathrm{er}}$ janvier 2011 (à la fois en termes relatifs et par rapport au reste de l'Espagne où aucune augmentation n'a été observée entre 2007 et 2010). 
Tableau 3. Régression logistique d'octroi de garde partagée après le divorce en Catalogne et ailleurs en Espagne (odds ratio)

\begin{tabular}{|l|l|c|c|}
\hline \multicolumn{1}{|c|}{ Variable / Catégories } & $\begin{array}{c}\text { Catalogne } \\
\text { Odds ratio }\end{array}$ & $\begin{array}{c}\text { Espagne hors Catalogne } \\
\text { Odds ratio }\end{array}$ & $\begin{array}{c}\text { Différence } \\
\text { (a) }\end{array}$ \\
\hline $\begin{array}{l}\text { Année de divorce } \\
2007\end{array}$ & 1 & 1 & \\
2008 & $1,154 * * *$ & $0,947 *$ & Oui \\
2009 & $1,201 * * *$ & $0,896 * * *$ & Oui \\
2010 & $1,438 * * *$ & $0,925 *$ & Oui \\
2011 (CCC) & $1,921 * * *$ & $1,074 * * *$ & Oui \\
2012 (CCC) & $2,722 * * *$ & $1,263 * * *$ & Oui \\
\hline Âge du père au divorce & 1 & 1 & \\
$<30$ ans & 1,129 & 1,003 & Non \\
$30-34$ ans & $1,282 * * *$ & 1,056 & Non \\
$35-39$ ans & $1,391 * * *$ & $1,171 * * *$ & Non \\
$40-44$ ans & $1,434 * * *$ & $1,256 * * *$ & Non \\
$45-49$ ans & $1,365 * * *$ & $1,361 * * *$ & Non \\
50 ans et + &
\end{tabular}

\begin{tabular}{|c|c|c|c|c|c|}
\hline \multirow{2}{*}{\multicolumn{6}{|c|}{ Différence d'âge }} \\
\hline & & & & & \\
\hline Même âge & 1 & & 1 & & \\
\hline Âge $F<$ âge $H$ & 0,955 & & 0,896 & $\star \star \star$ & Non \\
\hline Âge $F>$ âge $H$ & 0,976 & & 0,981 & & Non \\
\hline \multicolumn{6}{|l|}{ Nationalité } \\
\hline 2 Espagnols & 1 & & 1. & & \\
\hline 1 Espagnol et 1 étranger & 0,797 & $* * *$ & 1,056 & * & Oui \\
\hline 2 étrangers & 0,587 & $* * *$ & 0,899 & * & Oui \\
\hline \multicolumn{6}{|l|}{ Durée du mariage } \\
\hline$<5$ ans & 1 & & 1 & & \\
\hline $5-10$ ans & 1,123 & * & 1,116 & $\star * *$ & Non \\
\hline $10-20$ ans & 0,982 & & 1,167 & $* * *$ & Oui \\
\hline 20 ans et + & 0,915 & & 1,010 & & Non \\
\hline \multicolumn{6}{|c|}{ Situation du père avant le mariage } \\
\hline Célibataire & 1 & & 1 & & \\
\hline Divorcé/veuf & 0,867 & ** & 0,896 & $\star * *$ & Non \\
\hline \multicolumn{6}{|l|}{ Nombre d'enfants mineurs } \\
\hline 1 & 1 & & 1 & & \\
\hline 2 & 1,201 & $* \star \star$ & 1,115 & $\star \star \star$ & Non \\
\hline 3 et + & 1,093 & & 1,069 & & Non \\
\hline \multicolumn{6}{|c|}{ Procédure judiciaire } \\
\hline \multicolumn{6}{|l|}{ Requérant } \\
\hline Mari & 1 & & 1 & & \\
\hline Femme & 1,510 & $* * *$ & 1,527 & $* * *$ & Non \\
\hline Les deux & 1,512 & $* * *$ & 1,400 & $* * *$ & Non \\
\hline \multicolumn{6}{|l|}{ Séparation avant } \\
\hline Oui & 1 & & 1 & & \\
\hline Non & 1,371 & $* * *$ & 1,357 & $\star * *$ & Non \\
\hline
\end{tabular}


Tableau 3 (suite). Régression logistique d'octroi de garde partagée après le divorce en Catalogne et ailleurs en Espagne (odds ratio)

\begin{tabular}{|c|c|c|c|}
\hline Variable / Catégories & $\begin{array}{l}\text { Catalogne } \\
\text { Odds ratio }\end{array}$ & $\begin{array}{c}\text { Espagne hors Catalogne } \\
\text { Odds ratio }\end{array}$ & $\begin{array}{l}\text { Différence } \\
\text { (a) }\end{array}$ \\
\hline \multicolumn{4}{|l|}{ Durée du jugement } \\
\hline$<6$ mois & 1 & 1 & \\
\hline 6-11 mois & 0,952 & $0,941 * *$ & Non \\
\hline 12 mois et + & $0,827 * * *$ & $0,893 * * *$ & Non \\
\hline \multicolumn{4}{|l|}{ Jugement } \\
\hline $\begin{array}{l}\text { Divorce avec accord et } \\
\text { consentement mutuel }\end{array}$ & 1 & 1 & \\
\hline Divorce contesté & $0,627 * * *$ & $0,585 * * *$ & Non \\
\hline Constante & $0,065 * * *$ & $0,059 * * *$ & Non \\
\hline $\mathrm{R}^{2}$ de Nagelkerke & 0,06 & 0,03 & \\
\hline Effectif total & 40038 & 175599 & \\
\hline \multicolumn{4}{|c|}{$\begin{array}{l}\text { (a) « Non » signifie que les intervalles de confiance des rapports de risque se recoupent et que les variables n'ont } \\
\text { pas d'effet significativement différent en Catalogne et dans le reste de l'Espagne. } \\
\text { Note: Variable dépendante : garde partagée (oui vs. non). CCC : livre II du Code civil catalan entré en vigueur } \\
\text { le } 1^{\text {er }} \text { janvier } 2011 . ~ « 1 »: \text { modalité de référence. } \\
\text { Significativité }: * p<0,01 ; * * p<0,05 ;{ }^{* * *} p<0,1 \text {. } \\
\text { Source : Calculs des auteurs sur les données individuelles des jugements de divorce obtenues auprès de l'INE. }\end{array}$} \\
\hline
\end{tabular}

Figure 3. Odds ratio d'un accord de garde partagée après le divorce. Catalogne et Espagne à l'exclusion de la Catalogne. 2007-2012

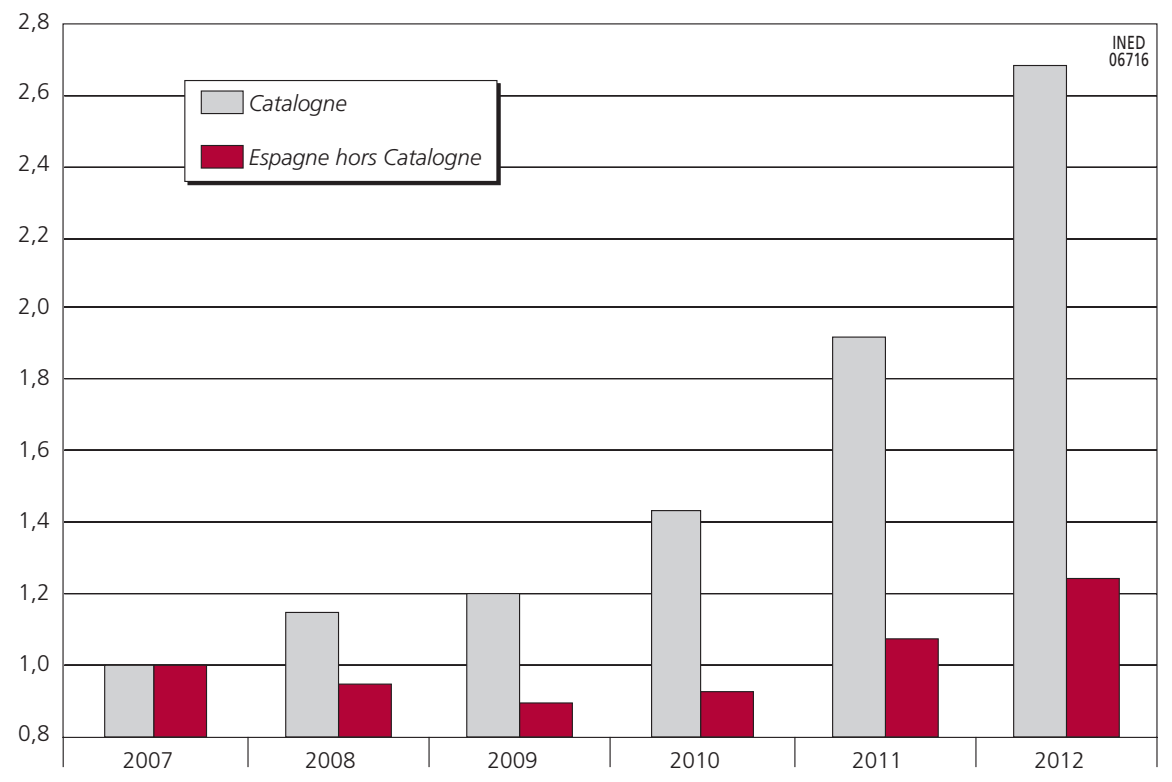

Note : Odds ratio après avoir pris en compte les variables de contrôles listées dans le tableau 3. L'année de référence est 2007 pour les deux territoires (odds ratio $=1$ ).

Source : Calculs des auteurs basés sur les données individuelles des jugements de divorce obtenues auprès de l'INE. 
de 84,4 \% lorsque la garde conjointe est attribuée contre 69,6\% lorsque la garde est attribuée à la mère et 59,7 \% lorsqu'elle est attribuée au père.

\section{Comparaisons internationales}

Lorsqu'on compare la Catalogne avec d'autres pays européens (tableau 4), on observe que la garde partagée est plus fréquente uniquement en Belgique, au Danemark et en Suède. Le cas de l'Italie est intéressant. Il y a eu dans ce pays, à peu près au même moment qu'en Espagne (en 2006), un changement de législation qui a facilité l'attribution de la garde conjointe, donnant ainsi aux enfants le droit d'avoir une relation équilibrée et durable avec leurs deux parents. Les tribunaux ont attribué l'autorité parentale conjointe dans un plus grand nombre de cas de séparation ou de divorce : de 15,4\% et 11,6 \% en 2005 à 89,8\% et 71,1\% en 2010 (De Blasio et Vuri, 2013) ${ }^{(17)}$. Cependant, cela reflète simplement les changements d'attribution de l'autorité parentale (legal custody).

Tableau 4. Proportion de gardes partagées d'enfants mineurs attribuées en Europe

\begin{tabular}{|l|c|c|c|c|}
\hline \multicolumn{1}{|c|}{ Pays } & $\begin{array}{c}\text { Proportion } \\
(\%)\end{array}$ & $\begin{array}{c}\text { Groupe d'âges } \\
\text { des enfants }\end{array}$ & Année & Source \\
\hline Catalogne & 30 & Tous & 2013 & www.ine.es \\
Espagne (sauf & 15 & Tous & 2013 & www.ine.es \\
\hline Catalogne) & 13 & Tous & 2008 & Vezzetti (2013) \\
\hline Allemagne & 1 & Tous & 2001 & Vezzetti (2013) \\
Autriche & 33 & Adolescents & $2006-2011$ & Sodermans et al. (2013) \\
Belgique & 39,22 & $7,11,15$ ans & Nés en 1995 & Vezzetti (2013) \\
Danemark & 19 & Tous & 2012 & Guillonneau et Moreau \\
France & 3 & Adolescents & $1998-2007$ & Vezzetti (2013) \\
Grèce & 5 & Tous & 2010 & Vezzetti (2013) \\
Italie & 20 & Tous & 2005 & Jensen (2005) \\
Norvège & 22 & Tous & 2013 & http://statline.cbs.nl/ \\
Pays-Bas & 3 & Tous & 2008 & Vezzetti (2013) \\
Portugal & 8 & Tous & 2011 & Vezzetti (2013) \\
République tchèque & $<1$ & Tous & 2009 & Vezzetti (2013) \\
Roumanie & 6 & Adolescents & 2009 & Bjarnason et Arnarsson \\
Royaume-Uni & 5 & Tous & 2011 & (2011) \\
Slovaquie & 35 & Tous & $2012-2013$ & Vezzetti (2013) \\
Suède & $<1$ & Tous & 2010 & Vezzetti (2013) \\
Suisse & & & &
\end{tabular}

(17) Alors qu'avant la réforme, les accords privilégiaient la garde exclusive de la mère et des droits de visite limités pour les pères, la nouvelle loi impose la garde conjointe, à moins que des circonstances très précises ne rendent la garde exclusive préférable. Ces circonstances doivent être consignées par écrit (provvedimento). Pour faciliter une relation étroite de l'enfant avec les deux parents, la loi introduit plusieurs dispositions financières relatives au paiement de la pension des enfants et à la localisation du foyer familial, mais ces dispositions ne semblent pas appliquées (De Blasio et Vuri, 2013; www.istat.it). 
Les arrangements financiers et organisationnels suivant la dissolution de l'union (physical custody) ont continué d'être basés sur les pratiques de garde exclusive : en 2010, près de $2 \%$ se voyaient attribuer une garde alternée et $3 \%$ une garde conjointe (Vezzetti, 2013).

\section{Conclusions}

Notre question initiale était : «Est-ce que le Code civil catalan de 2010 a joué un rôle dans le choix de garde partagée en Catalogne? ». La réponse est positive. La comparaison des tendances entre la Catalogne et le reste de l'Espagne confirme que la législation explique au moins en partie la progression de la garde partagée en Catalogne. En menant une analyse comparative de la prévalence de ce mode de garde durant la période précédant le CCC (2007-2010) avec la période suivante (2011-2012), nous pouvons résumer ces conclusions en trois points : les effets de la législation catalane (CCC) sur la réduction des inégalités entre les sexes dans la sphère familiale, la place de la Catalogne en Europe en matière de prévalence de la garde partagée; enfin nous consacrons quelques lignes aux limites de l'étude et à la nécessité de poursuivre les recherches dans ce domaine.

\section{Les effets du Code civil catalan}

Le CCC, créé et adopté en Catalogne par un gouvernement de gauche, est le produit d'une culture et d'une pratique judiciaire préexistantes à la réforme espagnole en 2005 et au CCC de 2010 dans la région autonome, associées à des comportements démographiques et à la situation socioéconomique de la région. Cela comprend une plus grande fréquence des divorces, particulièrement par consentement mutuel, ainsi qu'une plus forte occurrence de la garde partagée que dans d'autres régions d'Espagne. Par exemple, au cours des 15 dernières années, les divorces par consentement mutuel ont représenté $68 \%$ en moyenne entre 1999 et 2005 et $74 \%$ entre 2005 et 2012 en Catalogne, contre $56 \%$ et $64 \%$ pour les mêmes périodes dans le reste de l'Espagne. Le divorce par consentement mutuel est non seulement lié à une plus forte probabilité de garde partagée, mais il indique également que les parents divorcés ont une meilleure capacité à atteindre un consensus et à partager les pratiques parentales (Solsona et al., 2014). Ces éléments sont au cœur du nouveau CCC et sont plus particulièrement encouragés par l'introduction du plan parental.

Cependant, d'un point de vue sociologique, les pratiques de garde après le divorce doivent être replacées dans le contexte actuel de l'éducation et du soin apporté aux enfants. Les recherches sur les familles nucléaires ont montré que les pratiques quotidiennes de parentalité partagée des enfants mineurs en Catalogne gagnent progressivement du terrain sur la norme traditionnelle qui attribue cette responsabilité aux seules mères (Brullet, 2011). Les changements sont significatifs, mais modérés : 3 à 4 anciens couples mariés sur 10 comptant 
des enfants mineurs sont gérés au quotidien par le père et la mère, mais la répartition reste asymétrique pour le temps consacré et le type d'activités menées. Habituellement, ce sont les mères qui consacrent le plus de temps aux tâches domestiques et à l'éducation des enfants, malgré le fait que leur entrée sur le marché du travail mobilise un nombre d'heures équivalent à celui des pères. Bien qu'il faille reconnaître que les mères et les pères ne sont pas égaux dans la pratique parentale quotidienne, l'attribution croissante de la garde partagée en Catalogne suggère qu'un modèle de parentalité partagée émerge dans la société catalane et, avec lui, une transformation des identités de genre et des relations de pouvoir au sein de la famille. Il est important de noter que lorsque les époux acceptent le jugement de divorce, cela reflète non seulement un accord sur la décision du juge, mais aussi un consensus social sur les pratiques de parentalité après un divorce entre les acteurs du système judiciaire (avocats, procureurs, médiateurs, etc.) et les divorcés eux-mêmes.

Alors que la garde partagée constitue un bon instrument de socialisation pour l'enfant en promouvant l'égalité des sexes et en lui fournissant l'opportunité d'avoir des contacts avec ses deux parents, il existe des cas où la garde partagée n'est pas la meilleure option. Cela est reconnu par le CCC, qui établit spécifiquement que l'autorité judiciaire doit attribuer la garde à un seul parent si cela est dans l'intérêt de l'enfant (Picontó-Novales, 2012). Il existe deux exemples évidents dans lesquels ce cas de figure peut s'appliquer : si un parent a été poursuivi ou accusé pour des violences domestiques ou de genre ayant affecté directement ou indirectement des enfants mineurs (article 233.11.3 du CCC) et si un parent est négligent ou souffre de problèmes mentaux graves. Cependant, il existe d'autres situations où les parents divorcés ne doivent pas se voir confier des responsabilités égales pour les soins quotidiens. Par exemple, certains parents peuvent ne jamais avoir réellement participé à l'éducation de leur enfant ou ne pas en être capables (notamment à cause d'un handicap). Dans ces cas, la garde exclusive est de toute évidence préférable, ce qui doit être compris par les juges, les avocats, les travailleurs sociaux, les médiateurs, les psychologues et les autres professionnels impliqués dans les activités de conseil et de règlement des divorces (Bauserman, 2002).

Dans d'autres cas, la garde conjointe est demandée à cause d'une modification du jugement (relitigation) proposée par le mari en échange d'une réduction de pension si la garde exclusive a été attribuée à la mère. Ici, nous ne pouvons pas parler de consensus, mais d'une lutte de pouvoir constante entre les anciens partenaires.

Au cours d'un récent séminaire international (Espaces et temps dans les familles après le divorce $)^{(18)}$, des avocats et des chercheurs spécialisés en droit de la famille, des médiateurs et des divorcés ont pris la parole pour évoquer leurs expériences professionnelles et personnelles, et certains se sont inquiétés

(18) Espais i temps en les famílies del postdivorci, http://institutinfancia.cat/biblioteca/espais-i-tempsen-les-families-post-divorci-seminari-internacional-programa/ 
du fait que lors des premières années d'application du CCC, la garde partagée ait pu parfois être attribuée à tort. Si les données sur les jugements ne permettent pas d'examiner ce point en détail, il ne semble pas que la garde partagée soit l'option par défaut, dans la mesure où elle représente «seulement » un quart des cas.

À l'heure actuelle, la fréquence de la garde partagée en Catalogne est comparable à celle du Danemark, de la Suède et de la Belgique, loin devant les autres pays d'Europe.

\section{L'évolution future de la garde partagée}

Il n'est pas évident de prédire le niveau qu'atteindra la garde partagée, car cela dépend d'un grand nombre de facteurs qui comprennent des caractéristiques démographiques telles que l'âge au mariage, le fait d'avoir déjà connu une séparation, la nationalité ou le nombre d'enfants. La législation est également un facteur important. Les résultats comme ceux obtenus à partir du modèle ont montré que les facteurs démographiques et autres se comportent de manière très similaire en Catalogne et dans le reste de l'Espagne, alors que les niveaux absolus de garde conjointe en fonction des différentes caractéristiques sont constamment plus élevés en Catalogne (la principale exception étant la chance relativement plus faible pour les couples mixtes et étrangers de divorcés d'obtenir la garde partagée en Catalogne). Pourtant, le nouveau CCC ne peut expliquer à lui seul les différences territoriales, ces dernières étant déjà présentes avant son application au début de l'année 2011. Néanmoins, les lois particulières sur la famille en Catalogne semblent renforcer les différences observées. Il n'y a pas de signe de déclin ou de stabilité des attributions de garde conjointe en Catalogne, en particulier si on prend en compte les dernières données de l'Institut national de la statistique espagnol. La proportion a augmenté de 26,4\% à 29,6\% en Catalogne entre 2011 et 2013, et de 12,0\% à $15,3 \%$ dans le reste de l'Espagne.

\section{Limites de l'étude et pistes de futures recherches}

Bien que nos recherches éclairent la portée, les tendances et les caractéristiques démographiques fondamentales des modes de garde, elles ne portent que sur le moment du jugement de divorce, dans la mesure où les bulletins de jugement ne précisent pas s'il y a eu un changement juridique de l'accord. Par ailleurs, les données sur les jugements de l'INE ne divulguent aucune information du plan parental relative à la quantité de temps que chaque parent propose de passer avec l'enfant ou sur la quantité effective de temps qu'il lui consacre. En outre, la plupart des parents qui n'ont pas la garde ont également le droit de rendre visite à leur enfant de manière régulière, et les accords peuvent devenir plus fréquents à cause d'un changement d'emploi du temps du parent gardien ou suite à un souhait de l'enfant. Par conséquent, le temps réel passé avec les enfants peut être plus important pour des parents n'ayant pas la garde que pour des parents qui 
la partagent légalement. Comme ailleurs, la garde partagée en Catalogne ne correspond pas à une symétrie parfaite entre les deux parents en termes de tâches et de temps passé avec l'enfant, car cela est souvent irréalisable. En fait, le CCC n'a pas de définition précise du temps minimum que l'enfant doit passer avec chaque parent, bien que dans la pratique les enfants aient à vivre au moins un week-end sur deux et deux nuits par semaine avec le parent non gardien.

Étant donné l'importance croissante du phénomène et la manière dont il affecte chaque année des milliers de familles supplémentaires, les futures recherches doivent également analyser plus en profondeur les jugements en examinant les textes complets des accords de garde, des plans parentaux et des décisions des tribunaux en cas de contestation. De cette manière, nous serons mieux à même d'évaluer à quel moment la progression de la garde partagée revêt une véritable signification au regard de l'égalité des sexes.

Lautre limite des données sur les jugements réside dans l'impossibilité d'analyser les différences au sein de la population, en particulier les différences socioéconomiques, dans les accords de garde partagée. Nous savons par d'autres recherches que les parents disposant d'un statut socioéconomique plus élevé sont plus susceptibles d'opter pour une garde partagée après une séparation. Cela s'explique par le fait qu'ils disposent de plus de ressources et qu'ils ont plus de chances d'être les précurseurs de nouveaux comportements familiaux; ils peuvent aussi avoir des personnalités plus coopératives, des niveaux de conflit interparental moindres et être plus orientés sur les besoins de l'enfant en général (Turunen, 2015). Il serait intéressant d'un point de vue sociologique d'étudier des parents et des enfants avec des accords de garde et des situations économiques différentes après un divorce ou une séparation (d'un lien marital ou non marital) et de regarder comment ils ajustent les arrangements résidentiels avec le temps consacré aux soins des enfants, le travail salarié et le montant de l'aide financière - en particulier dans le contexte de crise économique qui a durement frappé l'Espagne ces dernières années. Pour une telle étude, il serait utile que les jugements de divorce ou les données d'études comprennent des informations sur les revenus des époux, leur niveau d'éducation et leur participation au marché du travail. Ces questions sont plus particulièrement intéressantes pour les mères. Par exemple, celles qui disposent de revenus plus élevés peuvent négocier une distribution plus équitable des tâches liées aux enfants, parce qu'elles ne dépendent pas autant (ou pas du tout) des paiements de pensions alimentaires. La garde conjointe semble donc tout à fait adaptée à leur situation. En outre, une proportion plus importante de femmes catalanes disposent d'un meilleur niveau d'éducation et occupent des emplois plus rémunérateurs que les femmes dans le reste de l'Espagne (selon les recensements de 2001 et 2011; INE). Les facteurs socioéconomiques peuvent très probablement expliquer au moins en partie les différences territoriales observées en matière de garde partagée. 
Remerciements : Cette étude est le résultat du projet $\mathrm{R}+\mathrm{D}+\mathrm{I}$ « Espacios de vida y usos del tiempo de las familias postdivorcio »(CSO2012-39157) financé par le ministère espagnol de l'Économie et de la compétitivité dirigé par Dr Solsona. Pour le Dr Spijker, l'étude a également bénéficié du soutien financier du programme «Ramón y Cajal » (RYC-2013-14851). Nous souhaitons également exprimer notre gratitude à Cristina Brullet, Marc Ajenjo et Ana Maria Goldani. 


\section{RÉFÉRENCES}

ARCE Ramón, FARIÑa Francisca, SEIJDO Dolores, 2005, « Razonamientos judiciales en procesos de separación: análisis cognitivo y de contenido de las motivaciones ", Psicothema, 17(1), p. 57-63.

BAUMAn Zygmunt, 2003, Liquid Love: On the Frailty of Human Bonds, Cambridge, Wiley, Polity Press, $176 \mathrm{p}$.

BAUman Zygmunt, 2005, Liquid Life, Cambridge, Polity Press, 224 p.

BAUSERMAN Robert, 2002, «Child adjustment in joint-custody versus sole-custody arrangements: A meta-analytic review », Journal of Family Psychology, 16(1), p. 91-102.

BAUSERMAN Robert, 2012, «A meta-analysis of parental satisfaction, adjustment, and conflict in joint custody and sole custody following divorce ", Journal of Divorce $E$ Remarriage, 53(6), p. 464-488.

BECK Ulrich, BECK-GERNSHEIM Elisabeth, 1995, The Normal Chaos of Love, Cambridge, Polity Press, 240 p.

BJARNASON Thoroddur, ARNARSSON Arsaell M., 2011, « Joint physical custody and communication with parents: A cross-national study of children in 36 Western countries ", Journal of Comparative Family Studies, 42(6), p. 871-890.

BRULLET Cristina, 2011, « La cura a la vida quotidiana a Catalunya: famílies i serveis. Balanç 2000-2010: petita infància i altres persones dependents », in Masjuan J. M. (ed.), Societat Catalana 2011, Barcelona, Associació Catalana de Sociologia, p. 127-177.

BuEHLER Cheryl, Gerard Jean M., 1995, « Divorce law in the United States: A focus on child custody », Family Relations, 44(4), p. 439-458.

CABRÉ Pla Anna, 1999, El sistema català de reproducció. Cent anys de singularitat demogràfica, Barcelona, Edicions Proa, 304 p.

CAnCiAn Maria, MeYer Daniel R., 1998, « Who gets custody? », Demography, 35(2), p. 147-157.

CATALÁN-FríAS M. José, 2011, « La custodia compartida », Revista Derecho y Criminología, Anales 2011(1), p. 57-82.

Cheadle Jacob E., Amato Paul R., King Valerie, 2010, « Patterns of nonresident father contact ", Demography, 47(1), p. 205-225.

DE Blasio Guido, VURI Daniela, 2013, « Joint custody in the Italian courts », IZA Discussion paper, $\mathrm{n}^{\circ} 7472$.

ESCOBEDO Anna, FlaQUer Lluís, NAVARRo-VARAS Lara, 2011, « The social politics of fatherhood in Spain and France: A comparative analysis of parental leave and shared residence », Ethnologie française, 42(1), p. 117-126.

FOLBERG Jay, 1991, Joint Custody and Shared Parenting, New York, Guilford Press, 380 p. GIDDENS Anthony, 1991, Modernity and Self-Identity. Self and Society in the Late Modern Age, Cambridge, Polity Press, 256 p.

GRAVERSEN Jørgen, 1986, « Denmark: Custody reform », Journal of Family Law, 25, p. 81-89. 
GuillonneAu Maud, MOREAU Caroline, 2013, « La résidence des enfants de parents séparés. De la demande des parents à la décision du juge. Exploitation des décisions définitives reçues par les juges aux affaires familiales au cours de la période comprise entre le 4 juin et le 15 juin 2012 », Paris, Ministère de la Justice.

HALlA Martin, 2009, "The effect of joint custody on marriage and divorce », IZA Discussion paper, $\mathrm{n}^{\circ} 4314$.

IBANEZ-VALVERDE Vicente J, 2004, «El laberinto de la Custodia Compartida. Claroscuros de un solo nombre con varios significados », Buletín de Derecho de Familia, 4(40-41), p. 1-15.

JENSEN An-Margritt, 2005, « Barn som bor med far bor også med mor », Samfunnsspeilet, 19(2), p. 30-38.

Juby Heather, Le Bourdais Céline, MARCIL-GRATtON Nicole, 2005, « Sharing roles, sharing custody? Couples' characteristics and children's living arrangements at separation », Journal of Marriage and Family, 67(1), p. 157-172.

KAUfMAnN Jean-Claude, 1993, Sociologie du couple, Paris, Presses universitaires de France, $125 \mathrm{p}$.

KAUFMANN Jean-Claude, 2007, Agacements. Les petites guerres du couple, Paris, Armand Colin, $256 \mathrm{p}$.

KITTER ØD Ragni Hege, LYNGSTAD Jan, 2012, « Untraditional caring arrangements among parents living apart: The case of Norway ", Demographic Research, 27(5), p. 121-152.

LATHROP Fabiola, 2012, «Custodia compartida y corresponsabilidad parental. Aproximaciones jurídicas i sociològicas », La Ley, Revista Jurídica Española de Doctrina, Jurisprudencia y Legislación, 7206, p. 1-6.

LESTHAEGHE Ron, 1983, «A century of demographic and cultural change in Western Europe: An explanation of underlying dimensions », Population and Development Review, 9(3), p. 411-435.

MASARDO Alexander, 2009, « Managing shared residence in Britain and France: Questioning a default "primary carer" model $»$, in Rummery K., Greener I., Holden C. (eds.), Social Policy Review 21: Analysis and Debate in Social Policy, Bristol, Policy Press, p. 197-214.

MCCLAIN Lauren R., 2011, « Better parents, more stable partners: Union transitions among cohabiting parents », Journal of Marriage and Family, 73(5), p. 889-901.

MORAn Gloria M., 1995, « The Spanish system of church and state », Brigham Young University Law Review, 2(7), p. 535-553.

PICONTÓ-NOVALES Teresa, 2012, « The equality rights of parents and the protection of the best interests of the child after partnership breakdown in Spain », International Journal of Law, Policy and the Family, 26(3), p. 378-400.

SAVOLAINEN Matti, 1986, « Finland: More rights for children », Journal of Family Law, 25, p. 113-126.

SELTZER, Judith A., 1994, «Consequences of marital dissolution for children », Annual Review of Sociology, 20, p. 235-266.

Simó Carles, SOlSONA Montserrat, 2010, «El registro estadístico de las rupturas de unión en España: evaluación e ideas para avanzar desde la demografía ", Papers: Revista de Sociologia, 95(3), p. 609-632.

Simó Carles, Solsona Montserrat, Houle René, TReviño Rocío, 2000, « Els determinants sociodemogràfics de les ruptures d'unions a Catalunya », Revista Catalana de Sociologia, 12, p. 87-110.

SODERMANS An Katrien, KOENRAAD Matthijs, GRAY Swicegood, 2013, « Characteristics of joint physical custody families in Flanders », Demographic Research, 28(29), p. 821-848. 
SOLSONA Montserrat, 1997, "The second demographic transition from a gender perspective: The case of Catalonia », in Cosio-Zavala M. E. (ed.), Women's Status and Family Dynamics, Paris, Cicred, p. 171-190.

SOlsona Montserrat, BRUllet Cristina, SPIJKER Jeroen, 2014, « Coparentalitat i custòdia compartida a Catalunya », Documents d'Anàlisi Geogràfica, 60(2), p. 1-29.

SPIJKER Jeroen, 2012, "Trends in custody arrangements in Spain since the Divorce Reform of 2005 », Papers de demografía, 404, Bellaterra, Spain.

SPIJKER Jeroen,BLANES-LlORENS Amand, 2009, « Mortality in Catalonia in the context of the third, fourth and future phases of the epidemiological transition theory ", Demographic Research, 20(8), p. 129-168.

SPRUIJT Ed, DUINDAM Vincent, 2009, « Joint physical custody in the Netherlands and the well-being of children », Journal of Divorce E Remarriage, 51(1), p. 65-82.

STATISTICS SwEDEN, 2014, « Different families live in different ways - a survey on residence and support of children after a separation », Demographic Reports, (1), Örebro, Sweden.

TORnello Samantha L., EMERY Robert, Rowen Jenna, POTTER Daniel, OCKer Bailey, XU Yishan, 2013, « Overnight custody arrangements, attachment, and adjustment among very young children ", Journal of Marriage and Family, 75(4), p. 871-885.

TURUNEN Jani, 2015, «Shared physical custody and children's experience of stress ", Families and Societies Working paper, 24.

VEZZETTI Vittorio, 2013, "European children and the divorce of their parents », Colibri - European Platform for Joint Custody co-parenting and childhood.

ZÚÑIGA Ángeles, 2012, « La custodia compartida », Escritura pública, 78, p. 64-66. 
Montserrat SOLSONA, Jeroen SPIJKER • INFLUENCE DU CODE CIVIL CATALAN (2010) SUR LES DÉCISIONS DE GARDE PARTAGÉE. COMPARAISONS ENTRE LA CATALOGNE ET LE RESTE DE ESPAGNE

Cet article a pour objectif principal d'analyser dans quelle mesure le Code civil catalan de 2010 influence la garde partagée des enfants après séparation des parents en Catalogne et de déterminer pourquoi la garde partagée a plus que doublé en Catalogne entre 2007 et 2012, alors que cela n'a pas été le cas dans les autres régions d'Espagne. Il décrit tout d'abord la réforme sur le divorce de 2005 en Espagne et les articles du Code civil catalan de 2010 qui concernent la garde partagée. Ensuite, il analyse les motifs et les caractéristiques des accords de garde partagée d'enfants mineurs, tels qu'ils ont été décidés par les tribunaux, à l'aide de données individuelles de l'Institut national de la statistique espagnol sur « les jugements en matière de séparations, divorces et annulations " pour la période 2007-2012 en Catalogne et dans le reste de l'Espagne. La législation catalane explique partiellement les différences entre la Catalogne et le reste de l'Espagne sur la garde partagée, non parce qu'elle recherche spécifiquement ce type d'accord sur la résidence des enfants, mais parce qu'elle favorise le partage des responsabilités parentales par deux outils spécifiques: des critères clairs pour déterminer le régime et les modalités de l'exercice de la garde, et la mise en place du plan parental, pouvant ainsi permettre de réduire les inégalités entre les sexes dans la sphère familiale.

\section{Montserrat SOlsona, Jeroen SPIJKER • EFFECTS OF THE 2010 CIVIL COdE ON TRENDS IN Joint Physical Custody in Catalonia. A Comparison With the Rest of Spain}

This article examines whether the Catalan 2010 Civil Code affects trends in joint physical custody in Catalonia, and why joint physical custody more than doubled in Catalonia during 2007-2012, although not in other regions of Spain. It first summarizes the 2005 divorce reform in Spain and the 2010 Catalan Civil Code on joint physical custody. It then describes the patterns and characteristics of physical custody arrangements of minor children, as adjudicated by judges, based on micro data from the Spanish National Statistics Institute on "Decrees of separations, divorces and annulments" for the period 2007-2012 in both Catalonia and the rest of Spain. It concludes that the Catalan legislation partially explains these observed differences, not because it advocates joint physical custody per se, but because it encourages shared parenting through the use of two specific tools: clear criteria for determining the regime and form of child custody; and a parental plan. Both of these elements also have great potential for reducing gender inequality in the family sphere.

\section{Montserrat SOLSONA, Jeroen SPIJKER • INFLUENCIA DEL CódIGO CIVIL (2010) SOBRE LAS DECISIONES DE CUSTODIA COMPARTIDA DE LOS PADRES DIVORCIADOS EN CATALUÑA. UNA COMPARACIÓn CON ESPAÑA}

Este artículo tiene por objetivo analizar en qué medida el código civil catalán (2010) influencia las decisiones de custodia compartida de los hijos después de la separación. Se analiza igualmente por qué motivos este tipo de custodia ha aumentado en dicha región más del doble entre 2007 y 2012, mientras que no ha sido así en otras regiones de España. Se presentan primero la reforma del divorcio de 2005 en España y los artículos del código civil catalán de 2010 que conciernen la custodia compartida. Se analizan después los motivos y las características de los acuerdos de custodia compartida decididos por los tribunales, gracias a los datos individuales provenientes del Instituto Nacional de Estadística sobre "los juicios en materia de separaciones, divorcios y anulaciones" durante el periodo 2007-2012. La legislación catalana explica en parte las diferencias entre Cataluña y el resto de España sobre la custodia compartida. No porque dicha legislación busque específicamente este tipo de acuerdo, sino porque favorece el reparto de las responsabilidades parentales gracias a dos instrumentos: claridad en los criterios para determinar el régimen y las modalidades de ejercicio de la custodia; establecimiento de un "plan parental" susceptible de reducir las desigualdades entre los sexos en la esfera familiar.

Mots-clés : Rupture de mariage, divorce, coparentalité, garde partagée, résidence alternée, égalité des sexes, Espagne, Catalogne.

Keywords: Marriage breakdown, divorce, co-parenting, shared physical custody, demography, gender equity.

Traduit par Camille Richou. 